\title{
ON DEGREE SETS AND THE MINIMUM ORDERS IN BIPARTITE GRAPHS
}

\author{
Y. MANOUSSAKIS \\ Université Paris XI (Orsay) \\ L.R.I., Bât. 490, F-91405 \\ Orsay Cédex, France \\ e-mail: yannis@lri.fr \\ AND \\ H.P. PATIL ${ }^{1}$ \\ Department of Mathematics \\ Pondicherry University \\ Puducherry, India \\ e-mail: hpppondy@gmail.com
}

\begin{abstract}
For any simple graph $G$, let $D(G)$ denote the degree set $\left\{\operatorname{deg}_{G}(v): v \in\right.$ $V(G)\}$. Let $S$ be a finite, nonempty set of positive integers. In this paper, we first determine the families of graphs $G$ which are unicyclic, bipartite satisfying $D(G)=S$, and further obtain the graphs of minimum orders in such families. More general, for a given pair $(S, T)$ of finite, nonempty sets of positive integers of the same cardinality, it is shown that there exists a bipartite graph $B(X, Y)$ such that $D(X)=S, D(Y)=T$ and the minimum orders of different types are obtained for such graphs.
\end{abstract}

Keywords: degree sets, unicyclic graphs.

2010 Mathematics Subject Classification: 05C07.

\section{REFERENCES}

[1] F. Harary, Graph Theory (Addison-Wesley, Reading Mass, 1969).

[2] S.F. Kapoor, A.D. Polimeni and C.W. Wall, Degree sets for graphs, Fund. Math. XCV (1977) 189-194.

\footnotetext{
${ }^{1}$ Research supported by Government of France.
} 
[3] Y. Manoussakis, H.P. Patil and V. Sankar, Further results on degree sets for graphs, AKCE Int. J. Graphs Comb. 1 (2004) 77-82.

[4] Y. Manoussakis and H.P. Patil, Bipartite graphs and their degree sets, R.C. Bose Centenary Symposium on Discrete Mathematics and Applications, (Kolkata India, 15-21 Dec. 2002), Electron. Notes Discrete Math. 15 (2003) 125.

doi:10.1016/S1571-0653(04)00554-2

[5] S. Pirzada, T.A. Naikoo, and F.A. Dar, Degree sets in bipartite and 3-partite graphs, Orient. J. Math Sciences 1 (2007) 39-45.

[6] A. Tripathi and S. Vijay, On the least size of a graph with a given degree set, Discrete Appl. Math. 154 (2006) 2530-2536.

doi:10.1016/j.dam.2006.04.003

Received 25 April 2011

Revised 29 April 2013

Accepted 29 April 2013 